\title{
Inversor de Tensão Utilizando Transformador de \\ Alta Freqüência com Modulação PWM
}

\section{Voltage Inverter Using High Frequency Transformer \\ with PWM Modulation}

\section{Gerson Osviani ${ }^{1}$; Ana Paula Bolognini²; Carlos Henrique Gonçalves Treviso ${ }^{3}$}

Resumo

\begin{abstract}
Este artigo apresenta uma nova topologia de inversor de freqüência formado por quatro conversores forward em paralelo. Estes conversores apresentam uma modificação para evitar que um circuito interfira no funcionamento do outro. A potência resultante é de $800 \mathrm{~W}$, com freqüência de chaveamento de $20 \mathrm{kHz}$. É apresentada uma completa análise das etapas de operação, bem como os procedimentos de projeto para a correta operação dessa topologia. Resultados de simulação, que correspondem ao funcionamento completo do circuito, são apresentadas para validar a análise do sistema.
\end{abstract}

Palavras-chave: Inversor. Conversor forward. Modulação PWM. Alta freqüência.

\begin{abstract}
This paper introduces a new frequency inverter topology with four forward converters, in a parallel topology configuration. These converters were modified to prevent one circuit to interfere on the functioning of other The resulting power is $800 \mathrm{~W}$, with $20 \mathrm{kHz}$ switch frequency. A complete analysis of the stages and procedures for the correct operation of the topology is presented. Simulation results, which correspond to the complete functioning of the circuit, are also presented, to validate the analysis of the system.
\end{abstract}

Key words: Inverter. Forward converter.; PWM Modulator. High frequency.

\section{Introdução}

Os conversores DC/DC podem ser definidos como circuitos que transformam uma determinada tensão contínua em outra também contínua.

O processo de transformação se dá por meio do chaveamento de transformadores compostos de núcleos magnéticos em altas freqüências. A saída do transformador é sempre proporcional à entrada.

Um exemplo simples é apresentado na Figura 1. Neste circuito, uma chave é acionada fechando a malha e transferindo a tensão para a o enrolamento primário do transformador. A tensão de saída aparece no enrolamento secundário.

1 Engenheiro Eletricista graduado na Universidade Estadual de Londrina.

2 Mestranda em Engenharia Elétrica na Universidade Estadual de Londrina.

3 Mestre e Doutor em Engenharia Elétrica (área de Sistemas de Energia) respectivamente em 1994 e 1999, pela Universidade Federal de Uberlândia. Docente do Departamento de Engenharia Elétrica na Universidade Estadual de Londrina. E-mail: treviso@uel.br 
Durante o período que a chave está fechada o núcleo é magnetizado. A desmagnetização do mesmo é feita enquanto a chave está aberta, pelo enrolamento de desmagnetização.

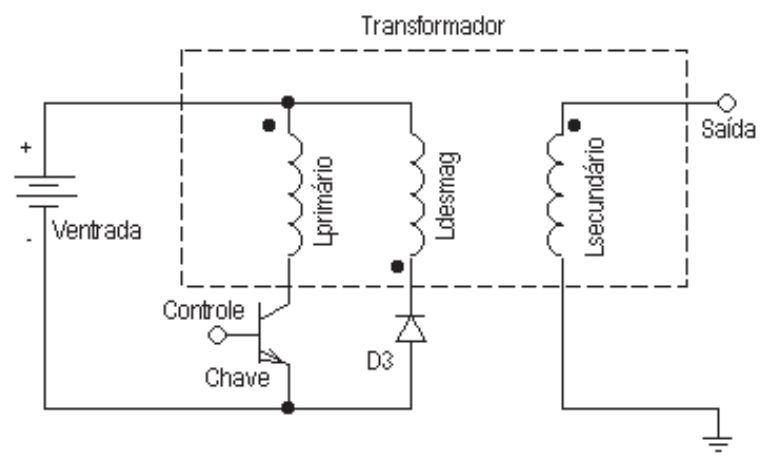

Figura 1. Circuito de um Conversor DC/DC

O sinal que aciona a chave da Figura 1 é modulado em largura de pulso (PWM - Pulse Width Modulation). Ou seja, quanto maior a largura do pulso que aciona a chave, maior a transferência de energia para a carga presente no secundário.

Para evitar que o sinal de saída seja uma onda quadrada semelhante à PWM de acionamento da chave, são adicionados ao circuito da Figura 1 alguns componentes com a função de filtrar a componente AC do sinal de saída do transformador, gerando uma saída DC ou semelhante a uma dada referência.

A Figura 2 traz o circuito completo do conversor DC como exemplo. É um forward com transformador.
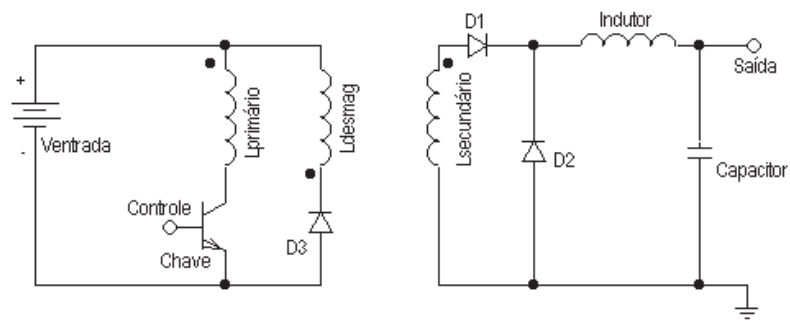

Figura 2. Circuito completo de um conversor DC/DC

Um tipo de conversor DC/AC muito utilizado é o inversor de tensão. A partir de uma tensão de entrada contínua é gerada uma saída alternada. $\mathrm{O}$ conversor é composto por conversores DC/DC controlados por PWM para obter diferentes formas de onda na saída.

A saída pode ser um sinal quadrado, triangular, senoidal ou outro qualquer. A freqüência também pode ser de diversos valores. Estas características do circuito são definidas pela aplicação do circuito.

As principais aplicações dos inversores são NoBreaks, acionamento de motores e amplificadores de áudio.

Na maioria dos inversores são necessárias pelo menos quatro chaves para gerar a tensão alternada na saída. Quando são acionadas duas delas, a carga é conectada diretamente à fonte e quando são acionadas as demais chaves, a carga é conectada inversamente.

Quando o controle é feito por PWM, existe um tempo morto entre os pulsos. Este tempo morto permite o melhor controle da tensão de saída e também que esta seja variável segundo uma referência, controlando assim o valor médio e o valor rms.

A Figura 3 apresenta o circuito de um inversor. É um conversor full-bridge.

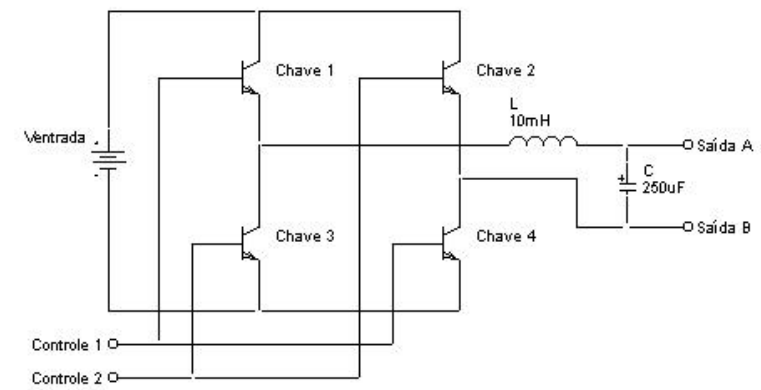

Figura 3. Inversor com quatro chaves

A geração dos sinais de controle das chaves é feita a partir da comparação de uma referência (sinal desejado na saída) e uma onda triangular. O resultado desta comparação é uma PWM que tem a freqüência da onda triangular e tem a modulação da largura de pulsos obedecendo à referência. No caso dos inversores, ainda é necessário que a PWM seja 
dividida entre as chaves. Ou seja, quando a referência é positiva, as chaves 1 e 4 recebem os pulsos, quando a referência é negativa, as chaves 2 e 3 recebem os pulsos.

Os resultados da simulação do circuito da Figura 3 são mostrados na Figura 4.

Os parâmetros para a simulação são:

- tensão de alimentação de $12 \mathrm{~V}$

- freqüência de chaveamento de $500 \mathrm{~Hz}$

- carga de saída de $100 \mathrm{~W}$

- onda senoidal como referência.

A Figura 4A mostra a onda senoidal usada como referência para o controle. A Figura 4B traz a referência invertida durante a parte negativa do ciclo e a triangular utilizada na comparação.

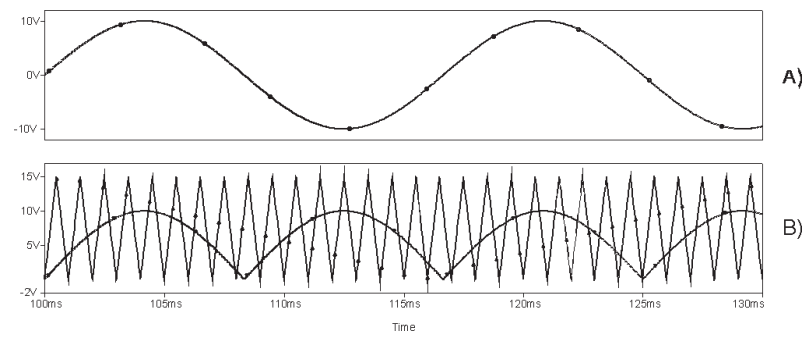

Figura 4. Referência senoidal e triangular

A Figura 5 traz os pulsos gerados para as chaves já separados. A Figura 5A mostra os pulsos que vão gerar os sinais positivos sobre a carga. A Figura 5B mostra os pulsos que vão gerar os sinais negativos.

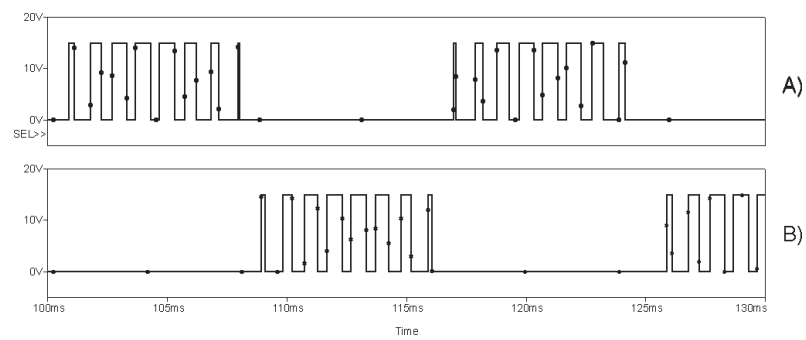

Figura 5. Pulso das chaves 1 e 4 e pulsos das chaves 2 e 3

A Figura 6 apresenta o sinal de saída resultante da simulação. Este sinal aparece sobre a carga, depois do filtro de saída, conforme mostrado na Figura 3.

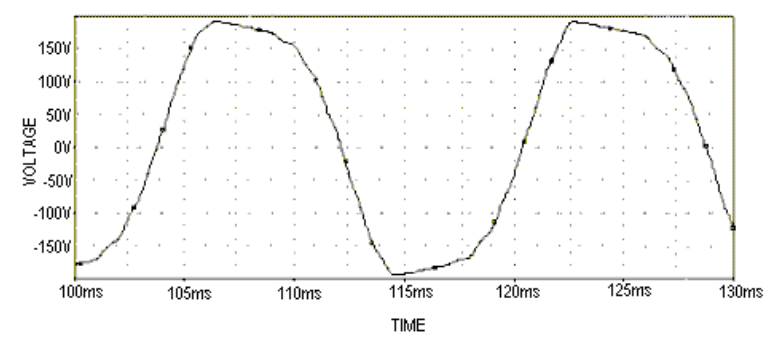

Figura 6. Saída do inversor

Uma aplicação dos inversores merece um realce: a alimentação de equipamentos com retificação a diodo na entrada, como por exemplo, o computador. Nesses aparelhos, a corrente de entrada possui picos que podem saturar o indutor do filtro de saída do inversor.

As simulações dos circuitos apresentados na Figura 7 servem para facilitar a compreensão desta situação. As fontes de alimentação são de $110 \mathrm{~V}$, as cargas são de mesma potência, aproximadamente $800 \mathrm{~W}$. O primeiro circuito apresenta apenas a carga resistiva conectada à saída do inversor. $\mathrm{O}$ segundo apresenta uma ponte completa, um capacitor para retificar a onda senoidal fornecida pela fonte e a carga resistiva. O valor do capacitor de $440 \mathrm{mF}$ foi escolhido considerando uma carga composta por duas fontes de computadores de $400 \mathrm{~W}$ com um capacitor de $220 \mathrm{mF}$ em cada uma.
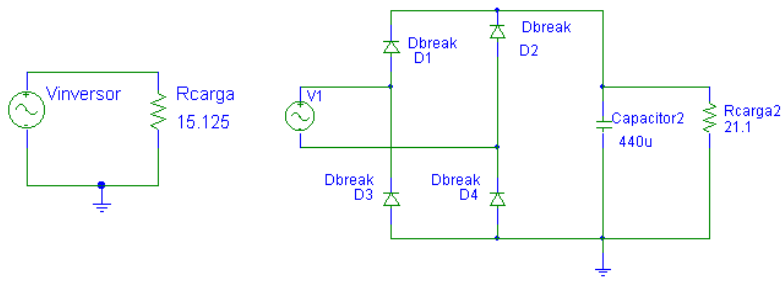

A) Figura 7. Uma carga resistiva e uma carga com ponte de diodo

Os resultados da simulação são apresentados na Figura 8. A Figura 8A mostra a corrente na fonte com apenas a carga resistiva. A Figura $8 \mathrm{~B}$ mostra a corrente na fonte com a ponte retificadora e a carga resistiva. 

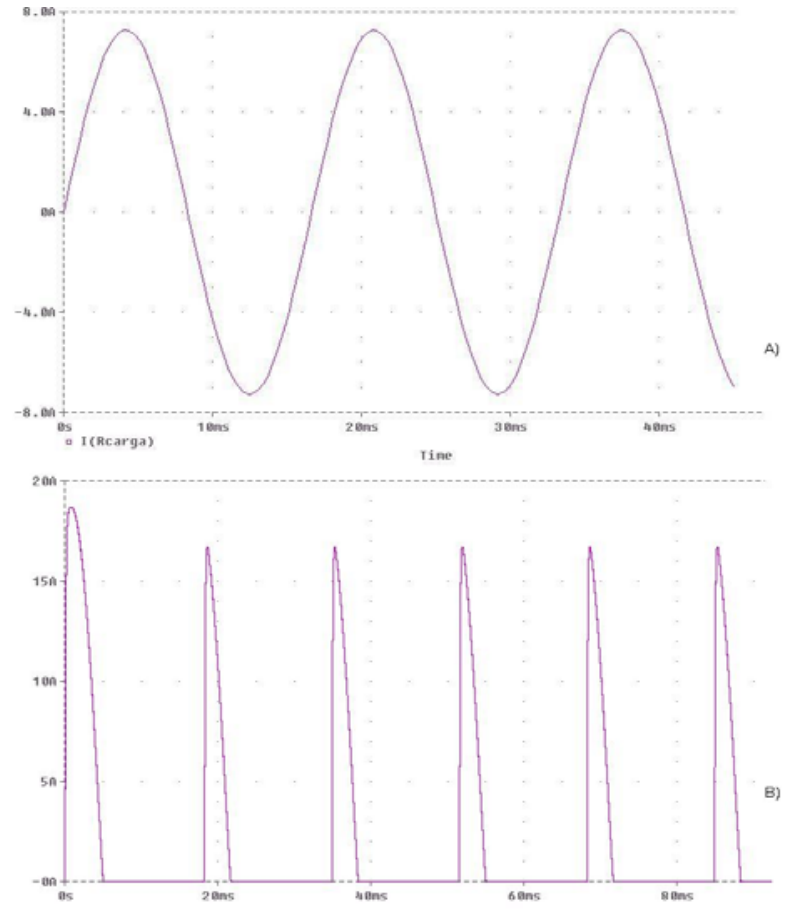

Figura 8. Correntes de saída

A simulação anterior mostrou que os picos de corrente na saída do inversor, com o uso de aparelhos com retificação em ponte completa na entrada, podem ser até 2,4 vezes maiores do que com o uso de uma carga resistiva.

Com o objetivo de obter o inversor de tensão, utilizando o transformador de alta freqüência, teremos como base o Conversor forward Convencional, como mostra a figura 9.

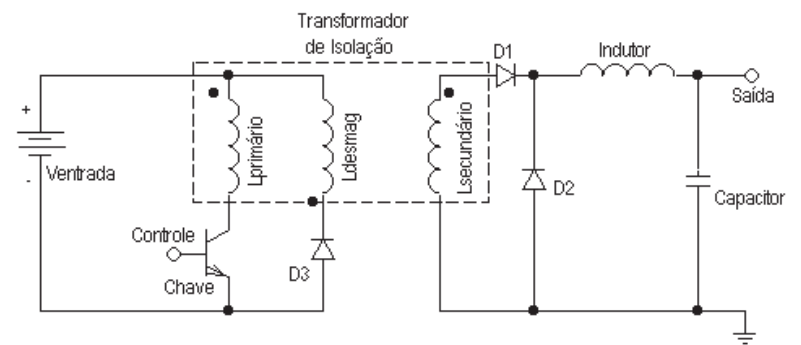

Figura 9. Conversor Forward

O conversor forward é composto por um transformador de isolação, uma chave, um filtro de saída, um diodo de desmagnetização, um diodo de retificação e um diodo de roda livre.
O transformador tem os enrolamentos primário, secundário e de desmagnetização. $\mathrm{O}$ enrolamento primário é conectado à fonte, o secundário é conectado à saída e o de desmagnetização serve para desmagnetizar o núcleo sobre a fonte.

O transformador de isolação pode ter os enrolamentos do primário e do secundário isolados. Isso garante maior segurança ao usuário em circuitos que trabalham com altas tensões no primário.

A chave é usada para produzir uma corrente pulsada no enrolamento primário do transformador, que é acionada por um circuito de controle que compara a saída do conversor com uma referência.

O filtro de saída filtra a componente alternada do sinal quadrado, que sai do secundário do transformador, dessa maneira, a carga recebe apenas uma tensão contínua (DC).

O diodo de desmagnetização evita que o enrolamento de desmagnetização tenha corrente durante o período em que a chave está acionada. O diodo retifica a saída do transformador evitando que a corrente do indutor atue sobre ele. $\mathrm{O}$ diodo de roda livre fornece o caminho para a corrente do indutor enquanto a chave está aberta.

No instante em que a chave é acionada, a tensão do enrolamento primário aparece no enrolamento secundário, multiplicado pela relação de espiras (n). A tensão do secundário vai produzir uma corrente através do diodo D1, carregar o indutor e o capacitor e fornecer energia para a carga. No enrolamento de desmagnetização aparece o valor da tensão da fonte, fazendo com que exista o dobro desta tensão sobre o diodo D3, caso essa relação seja de 1:1.

Quando a chave é aberta, as tensões nos enrolamentos do transformador invertem seus valores. Com isso, o enrolamento de desmagnetização vai atuar, desmagnetizando o transformador sobre a fonte. O diodo D1 vai bloquear, isolando o circuito do transformador do circuito do filtro de saída. Assim, o indutor e o capacitor suprirão a energia para a carga. E o caminho 
de corrente entre a carga e o indutor vai ser feito através do diodo D2.

Para a relação de transformação de 1:1 entre os enrolamentos do primário e de desmagnetização, o tempo de desmagnetização deve ser o mesmo que o de magnetização. Por isso, a máxima largura de pulso que este conversor suporta é 0,5. Qualquer valor acima deste provoca a saturação do transformador.

A relação de transformação é dada pela Equação 1.1 (MELLO, 1990).

$$
\mathrm{n}=\frac{\text { Nprimário }}{\text { N sec undário }}
$$

E a tensão no secundário é dada pela Equação 1.2 (MELLO, 1990).

$$
\text { V secundário }=\frac{\text { Vprimário }}{n}
$$

Usando D como a largura de pulso da PWM de acionamento da chave e VD1 como a tensão em D1, a tensão de saída sobre a carga é obtida pela Equação 1.3 (MELLO, 1990).

$$
\text { Vsaída }=\frac{\text { D.Vprimário }}{n}-\text { VD1 }
$$

As relações de largura de pulso máxima e mínima estão definidas na Equação 1.4 (MELLO, 1990).

$$
\mathrm{D} \min =\frac{\text { Ve min }}{\text { Vemáx }} . \text { Dmáx }
$$

O projeto do transformador inicia-se com a escolha do núcleo mais adequado.

As correntes presentes no transformador são a do primário (Ip), a do secundário (Is) e a de magnetização (Img). Obedecendo à relação de espiras do transformador, as correntes do primário e do secundário geram campos magnéticos que se anulam, como mostra a Equação 1.5 (MELLO, 1990).

$$
\text { Np.lp }=\text { Ns.ls }
$$

A energia armazenada em um núcleo magnético é dada pela Equação 1.6 (MELLO, 1990).

$$
E=\frac{1}{2} \cdot \text { L.I }{ }^{2} \quad(J)
$$

A corrente de magnetização pode saturar o núcleo, caso sua energia armazenada seja maior que a energia que o núcleo usado suporte. Para evitar isso, é necessário que a escolha do núcleo considere essa possibilidade.

A partir do cálculo do produto das áreas (Ap), é possível escolher o núcleo mais adequado. Então se calcula o Ap considerando a energia que será armazenada no núcleo, o que é feito com a Equação 1.7 (RASHID, 1993).

$$
A p=\left(\frac{2 \cdot E \cdot 10^{4}}{\text { ku.kj.Bmáx }}\right)^{z} \quad\left(\mathrm{~cm}^{4}\right)
$$

Onde:

$\mathrm{ku}$ - fator de utilização das janelas

$\mathrm{kj}$ - coeficiente de densidade de corrente nos fios

Bmáx - densidade máxima de fluxo (T)

$\mathrm{z}=\frac{1}{1-\mathrm{x}}-$ constante que depende do núcleo utilizado
$($ MELLO, 1990$)$.

Esta maneira de calcular o Ap não é utilizada para escolher o núcleo, porque suas variáveis somente serão definidas no final do projeto do transformador. Esta equação vai ser utilizada para confirmar a possibilidade de uso no núcleo escolhido.

No caso do conversor forward, a equação utilizada para o cálculo do Ap e a escolha do núcleo é a Equação 1.9 (MELLO, 1990). 


$$
A p=\left(\frac{2,65 \cdot P s \cdot 10^{4}}{\text { kj.B.f }}\right)^{z} \quad\left(\mathrm{~cm}^{4}\right)
$$

Onde:

Ps - potência de saída

f - freqüência de chaveamento

$\mathrm{B}=\frac{\text { Vemin }}{\text { Vemáx }}$.Bmáx $\begin{gathered}\text { densidade de fluxo (T) } \\ \text { 1990). }\end{gathered}$

Escolhido o núcleo, a próxima etapa é o cálculo de determinados parâmetros do transformador. $\mathrm{O}$ primeiro item é o número de espiras do primário e do secundário, obtido através da Equação 1.11 (MELLO, 1990).

$$
\mathrm{Np} \geq \frac{\text { Vemin.Dmáx }}{\text { Ae.B.f }}
$$

Onde:

Dmáx - largura de pulso máxima que o conversor permite, no caso do forward é 0,5

Ae - área efetiva do núcleo $\left(\mathrm{cm}^{2}\right)$.

As indutâncias do transformador (Lp e Ls) são dadas por meio da relação entre o número de espiras e o fator de indutância (Al), Equações 1.12 e 1.13 (MELLO, 1990). O valor do Al depende do núcleo utilizado e pode ser encontrado com o uso da Tabela em Mello, (1990).

$$
\begin{aligned}
& \mathrm{Lp}=\mathrm{Np}^{2} \cdot \mathrm{Al} \quad(\mathrm{H}) \\
& \mathrm{Ls}=\mathrm{Ns}^{2} \cdot \mathrm{Al} \quad(\mathrm{H})
\end{aligned}
$$

As correntes médias do primário e do secundário são obtidas pelas Equações 1.14 e 1.15 (MELLO, 1990), respectivamente, a partir da corrente de saída (Io). Esta corrente é definida nas especificações do projeto.

$$
\text { Is }=\left(10+\frac{\text { lo }}{10}\right) \cdot \text { Dmáx }
$$

$$
I p=\frac{I s}{n} \quad(A)
$$

A corrente de magnetização é obtida pela Equação 1.16 (MELLO, 1990).

$$
\operatorname{Img}=\frac{\text { Dmáx.Vemáx }}{\text { Lp.f }} \quad(A)
$$

O número de espiras do enrolamento de desmagnetização e a indutância de desmagnetização são equacionados de maneira semelhante ao enrolamento do primário. Isso porque a energia de magnetização é igual à energia de desmagnetização. Então o número de espiras do enrolamento de desmagnetização é igual ao número de espiras do enrolamento do primário.

Para o equacionamento dos fios, é necessário o cálculo da densidade de corrente $(\mathrm{J})$ para o núcleo usado. A densidade de corrente é obtida com a Equação 1.17 (MELLO, 1990).

$$
J=397 \cdot A p^{-0,12} \quad\left(A / \mathrm{cm}^{2}\right)
$$

A área de cobre de cada enrolamento será dada pela Equação 1.18 (MELLO, 1990).

$$
\text { Acu }=\frac{\text { leficaz }}{J}\left(\mathrm{~cm}^{2}\right)
$$

A condução de correntes alternadas em condutores de secção transversal produz o efeito pelicular ou "Skin". Quanto maior a freqüência, menor a profundidade de condução. Para evitar isso, são utilizados diversos fios em paralelo, considerando o raio do fio menor que a máxima profundidade de corrente para a freqüência de chaveamento usada.

A Figura 10 mostra a bitola do fio que pode ser utilizada pela freqüência de chaveamento. Qualquer valor acima da linha pode ser utilizado numa determinada freqüência, porque o raio é menor que a profundidade de corrente em uma dada freqüência (TREVISO, 1999). 


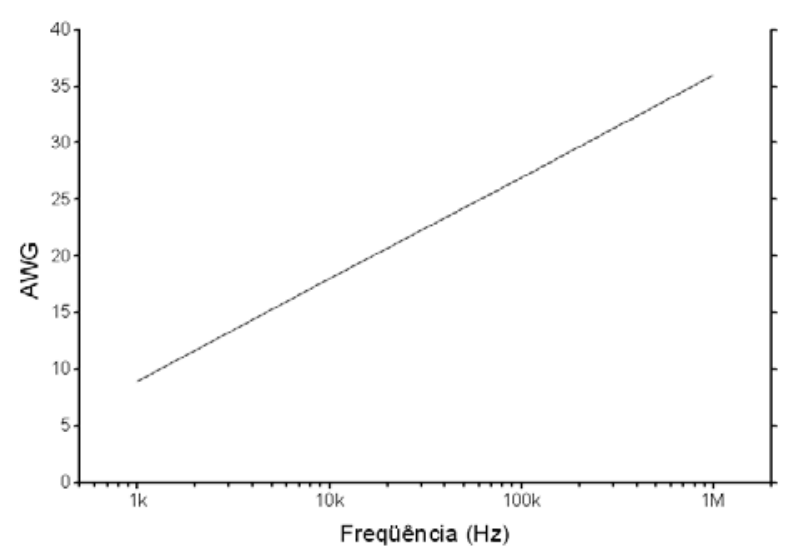

Figura 10. Fios usados pela freqüência de chaveamento

O filtro de saída serve, fundamentalmente para filtrar as componentes alternadas que saem do secundário do transformador. Conforme a freqüência de corte, ele obtém na saída uma fonte DC.

O filtro é composto de um indutor em série com a carga e um capacitor em paralelo com a mesma.

O projeto do filtro de saída será em função da freqüência de corte, dada pela Equação 1.19 (SEDRA; SMITH, 2000).

$$
\mathrm{fC}=\frac{1}{2 \pi \sqrt{\mathrm{LC}}}(\mathrm{Hz})
$$

Onde:

fc - freqüência de corte

$\mathrm{L}$ - valor do indutor

$\mathrm{C}$ - valor do capacitor.

A energia máxima é dada por.

$$
E=\frac{1}{2} \cdot L \cdot(I O)^{2} \quad(J)
$$

Com a energia obtida na Equação 1.20 (MELLO, 1990) e substituindo na Equação 1.7 (RASHID, 1993), obtém-se o Ap necessário para o indutor. E com ele, obtém-se o núcleo.

Definido a máxima energia que o indutor vai armazenar e o núcleo que vai ser utilizado, o próximo passo é o cálculo do Al que vai ser necessário no núcleo.
O Al é dado pela Equação 1.21 (MELLO, 1990).

$$
\mathrm{Al}=\frac{\mathrm{Ae}^{2} \cdot \mathrm{Bmáx}^{2}}{2 \cdot \mathrm{E}} \quad(\mathrm{H} / \mathrm{esp})
$$

Como o Al é uma característica do núcleo, ele só pode ser alterado adicionando ao caminho magnético um meio que diminua o Al. Esse meio é chamado de entreferro e é dado pela Equação 1.22 (MELLO, 1990).

$$
\lg =\frac{\mu_{0} \cdot A e}{2 \cdot A l} \quad(m)
$$

O número de espiras é dado pela Equação 1.23 (MELLO, 1990).

$$
N=\sqrt{\frac{L}{A l}}
$$

O dimensionamento dos fios é igual ao processo usado para os enrolamentos do transformador.

\section{A Nova Topologia Proposta para o Inversor}

A nova topologia de inversor de tensão será formada por quatro conversores forward, alimentados com tensão DC de $12 \mathrm{~V}$, com tensão de saída senoidal de $110 \mathrm{~V}$ eficaz e baixa Distorção Harmônica Total (THD - Total Harmonic Distortion).

A nova topologia de um inversor de tensão é formada por quatro conversores forward modificados, em paralelo, como mostrado na Figura 11. Esta topologia considera os conversores A e B como geradores de pulsos positivos na carga. A ligação em paralelo destes conversores ocasiona um ciclo ativo máximo (Dmáx) para a saída do circuito, ou seja, igual a 1,0. Enquanto um conversor está desmagnetizando o núcleo, o outro está fornecendo energia. $\mathrm{O}$ mesmo acontece com os conversores $\mathrm{C}$ e $\mathrm{D}$, em paralelo, que são geradores de pulsos negativos. As chaves utilizadas na Figura 12 são MOSFETs, e estas são usadas quando a freqüência 
de chaveamento é maior que $20 \mathrm{kHz}$. Elas são acionadas para permitir que os diodos conduzam nos instantes definidos, atuando como roda livre da corrente do filtro e da carga durante o tempo morto.

O sinal de acionamento das chaves é modulado em largura de pulso (PWM - Pulse Width Modulation). A potência do inversor será de $800 \mathrm{~W}$ com freqüência de chaveamento de $20 \mathrm{kHz}$.

Neste trabalho, é analisada a operação do conversor forward, mostrado na Figura $11 \mathrm{em}$ diagrama de blocos.

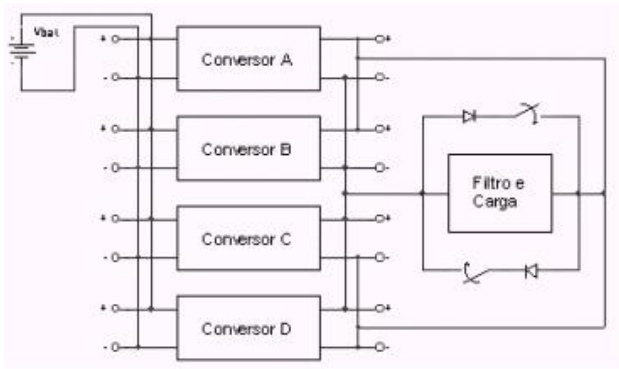

Figura 11. Nova topologia de inversor

As saídas dos quatro conversores são ligadas conforme a Figura 11. O uso de chaves no secundário é necessário para evitar que um conversor interfira no funcionamento dos demais.

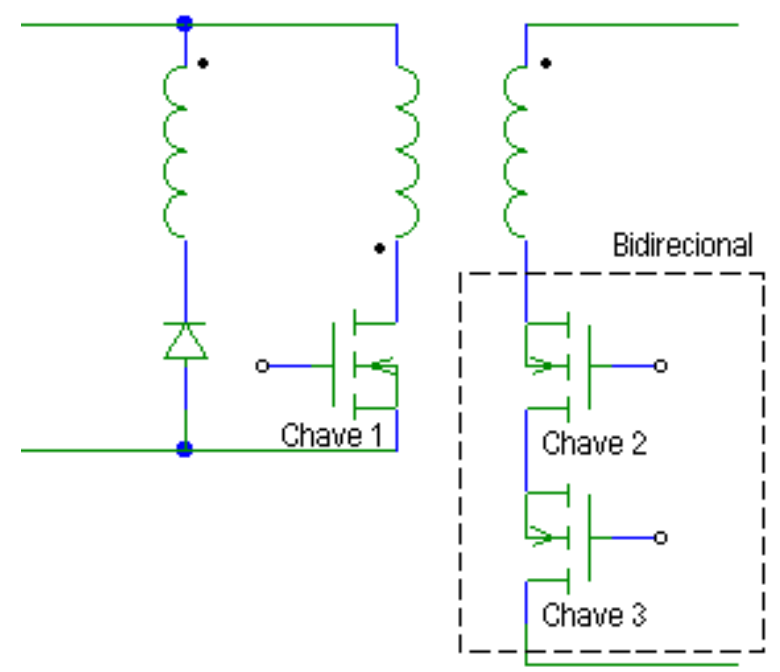

Figura 12. Circuito de cada inversor

As chaves 2 e 3 da Figura 12 isolam o secundário durante o período em que o conversor não está transferindo energia. Neste período, ele vai estar desmagnetizando o núcleo através do enrolamento de desmagnetização e os sinais provenientes dos demais conversores sobre o enrolamento do secundário podem interferir.

Como cada chave possui um diodo intrínseco permitindo condução do source para o drain, é necessário o uso da chave 3 para evitar a condução de corrente pela chave 2 durante os períodos em que a saída atinge valores negativos.

Os tempos de condução das chaves 1, 2 e 3 são iguais e em fase.

Os diodos servem para a roda livre da corrente do filtro e da carga durante o tempo morto. As chaves são MOSFETs que serão acionados para permitir que os diodos conduzam nos instantes definidos.

A Figura 13 mostra a ligação dos diodos de roda livre com a chave ligada em série. Ela permite o controle sobre os tempos de condução do diodo.

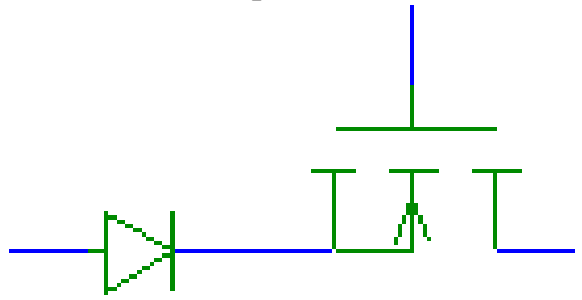

Figura 13. Diodos de Roda Livre

A Figura 14 apresenta os componentes de cada conversor no circuito de potência da nova topologia apresentada.

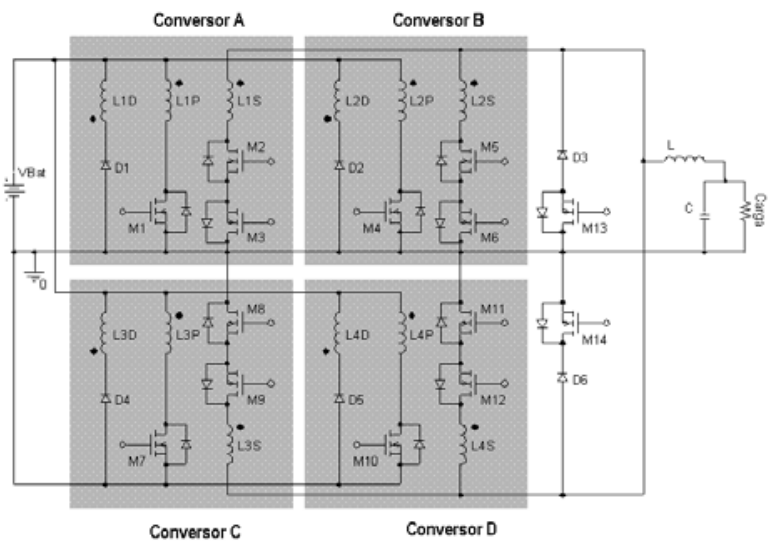

Figura 14. Cada conversor 
Uma característica deste conversor é a bidirecionalidade de transferência de energia entre a fonte e a carga. Quando são utilizadas cargas não resistivas, existem as potências reativas que são entregues à carga e devolvidas à fonte durante as etapas de funcionamento.

\section{Procedimento Proposto}

Um período de funcionamento do inversor pode ser dividido em dez etapas distintas.

Tabela 1. Descrição das etapas de funcionamento do conversor

\begin{tabular}{c|l}
\hline Etapa & Descrição \\
\hline $\mathbf{1}$ & Transferência de energia para a carga com conversor A \\
\hline $\mathbf{2}$ & Transferência de energia para a carga com conversor B \\
\hline $\mathbf{3}$ & Descarga de energia através do conversor C \\
\hline $\mathbf{4}$ & Descarga de energia através do conversor D \\
\hline $\mathbf{5}$ & Transferência de energia para a carga com conversor C \\
\hline $\mathbf{6}$ & Transferência de energia para a carga com conversor D \\
\hline $\mathbf{7}$ & Descarga de energia através do conversor A \\
\hline $\mathbf{8}$ & Descarga de energia através do conversor B \\
\hline $\mathbf{9}$ & Tempo morto entre os conversores A e B \\
\hline $\mathbf{1 0}$ & Tempo morto entre os conversores C e D \\
\hline
\end{tabular}

No secundário dos conversores está conectada uma carga não resistiva, formada pelo filtro de saída mais a carga. Por isso existem ciclos de transferência de energia para a carga e ciclos de descarga da carga na fonte.

O tempo morto é o período em que não há transferência de energia e é usado o diodo de roda livre para manter a corrente armazenada no indutor.

A Figura 15 mostra as etapas de funcionamento do conversor referenciadas na Tabela 1 .

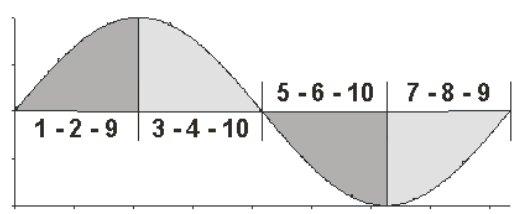

Figura 15. Etapas de funcionamento do conversor

A Figura 16 apresenta a carta de tempos da comutação dos transistores. Como pode ser observado, a chave M13 é complementar das chaves M1, M2, M3, M4, M5 e M6; e a chave M14 é complementar das chaves M7, M8, M9, M10, M11 e M12.

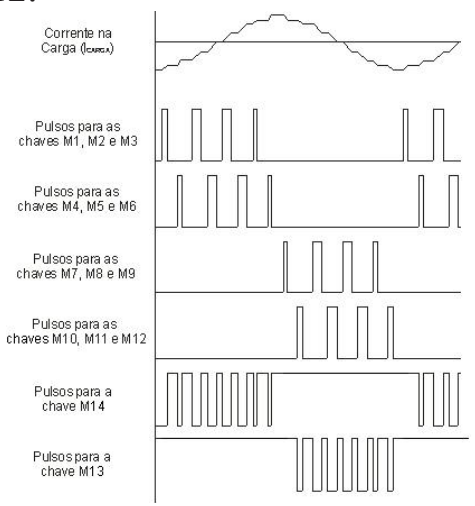

Figura 16. Formas de onda

A linha em negrito indica o caminho das correntes para a respectiva etapa:

Primeira Etapa: transferência de energia para a carga com conversor A: as chaves M1, M2 e M3 são acionadas para que o conversor A transfira energia para a carga. Neste instante, os demais conversores estão desacoplados, livres para que desmagnetizem seus núcleos, caso seja necessário.

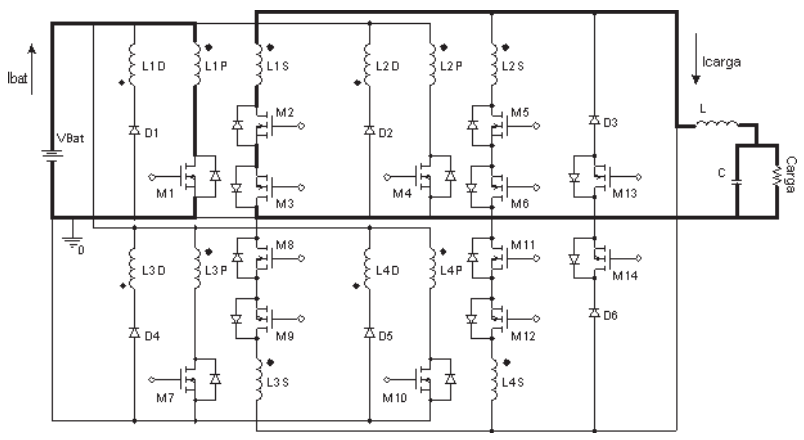

Figura 17. Primeira Etapa

Segunda Etapa: transferência de energia para a carga com conversor B: quando as chaves M4, M5 e M6 são acionadas para que o conversor B transfira energia para a carga. 


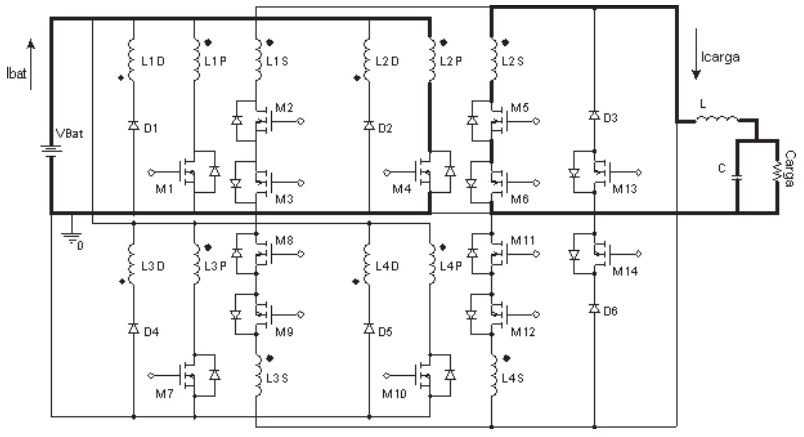

Figura 18. Segunda Etapa

Terceira Etapa: descarga de energia através do conversor C: as chaves M7, M8 e M9 são acionadas para que o conversor $\mathrm{C}$ transfira energia para a carga. Mas, como o indutor ainda está carregado por causa da etapa anterior, a transferência de energia é feita da carga para a fonte através do conversor $\mathrm{C}$.

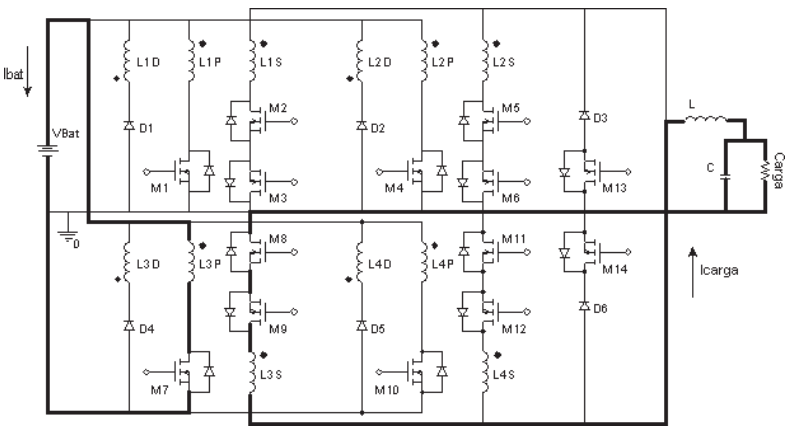

Figura 19. Terceira Etapa

Quarta Etapa: descarga de energia através do conversor D: as chaves M10, M11 e M12 são acionadas para descarregar a carga na fonte pelo conversor D.

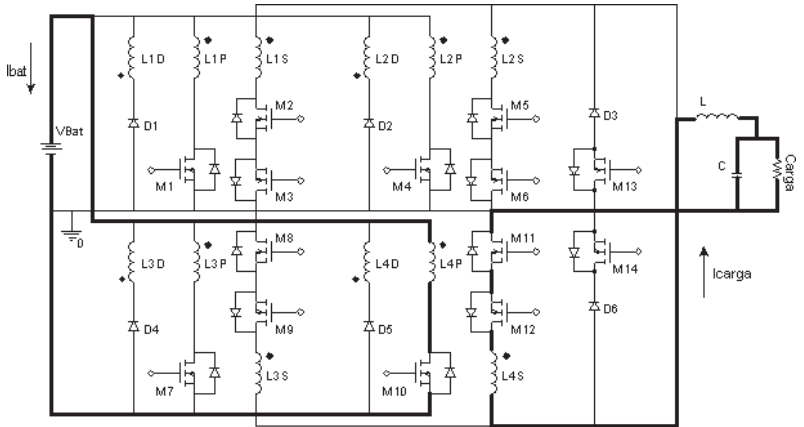

Quinta Etapa: transferência de energia para a carga com conversor C: a carga já está descarregada e as chaves M7, M8 e M9 são acionadas para que o conversor $\mathrm{C}$ transfira energia para a carga. É semelhante à etapa 1 , porém, agora, com sinal negativo de tensão sobre a carga.

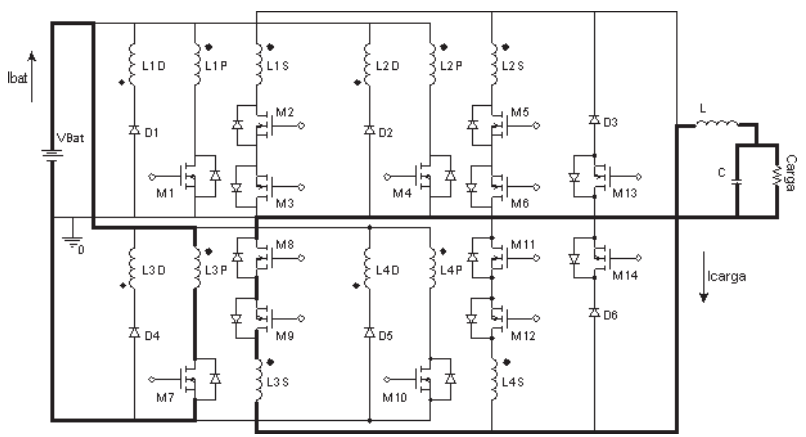

Figura 21. Quinta Etapa

Sexta Etapa: transferência de energia para a carga com conversor D: as chaves M10, M11 e M12 são acionadas para que o conversor D transfira energia para a carga.

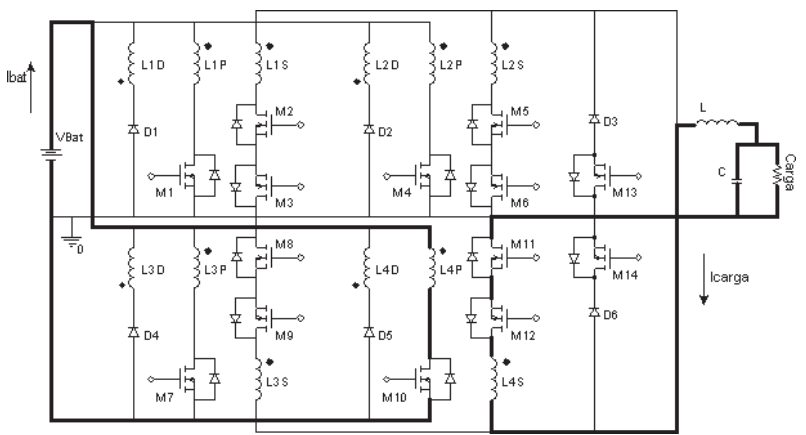

Figura 22. Sexta Etapa

Sétima Etapa: descarga de energia através do conversor A: as chaves M1, M2 e M3 são acionadas para que o conversor A transfira energia para a carga. Mas, como o indutor ainda está carregado por causa da etapa anterior, a transferência é feita da carga para a fonte através do conversor A.

Figura 20. Quarta Etapa 


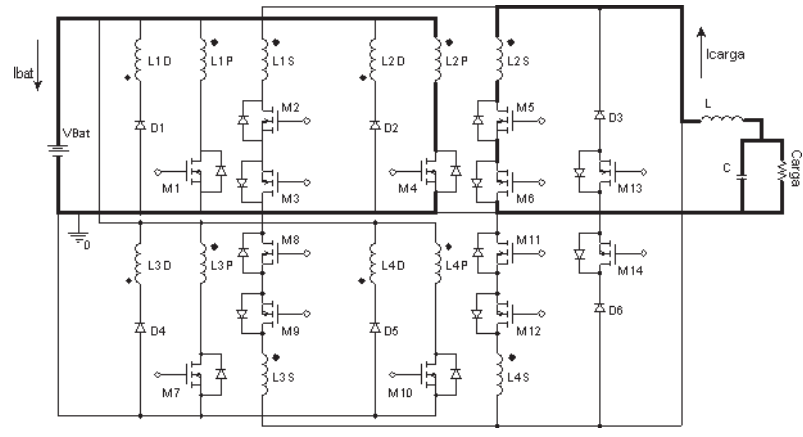

Figura 23. Sétima Etapa

Oitava Etapa: descarga de energia através do conversor B: as chaves M4, M5 e M6 são acionadas para descarregar a carga na fonte pelo conversor B.

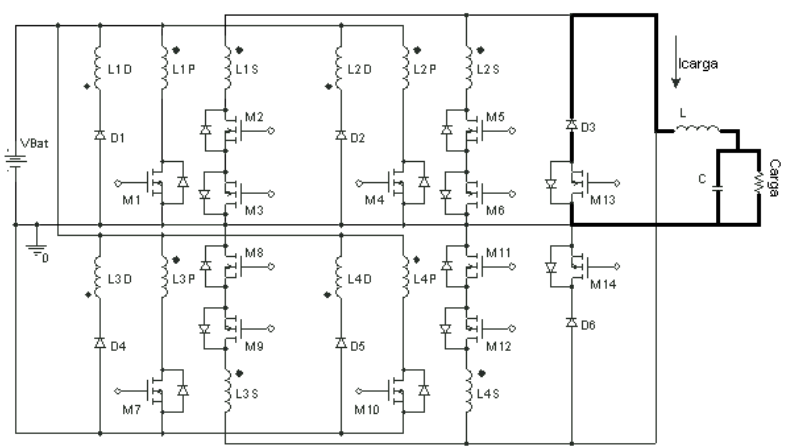

Figura 24. Oitava Etapa

Nona Etapa: tempo morto entre as chaves A e B: a chave M13 é acionada fornecendo um caminho para a corrente $\mathrm{I}_{\mathrm{CARGA}}$, durante o tempo entre o acionamento dos conversores A e B. O controle deve garantir que a chave M13 não seja acionada durante as etapas 3, 4, 5 e 6 e nos intervalos delas. Isso provocaria um curto circuito na saída dos conversores $\mathrm{C}$ e D.

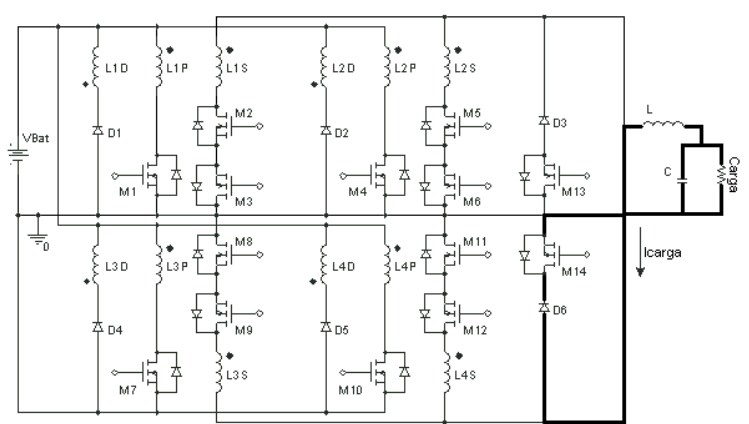

Décima Etapa: tempo morto entre as chaves C e D: a chave M14 é acionada fornecendo um caminho para a corrente $\mathrm{I}_{\mathrm{CARGA}}$ durante o tempo entre o acionamento dos conversores $\mathrm{C}$ e $\mathrm{D}$. O não acionamento da chave M14 deve ser garantido durante as etapas 1, 2, 7 e 8 e entre elas, para evitar um curto circuito na saída dos conversores A e B.

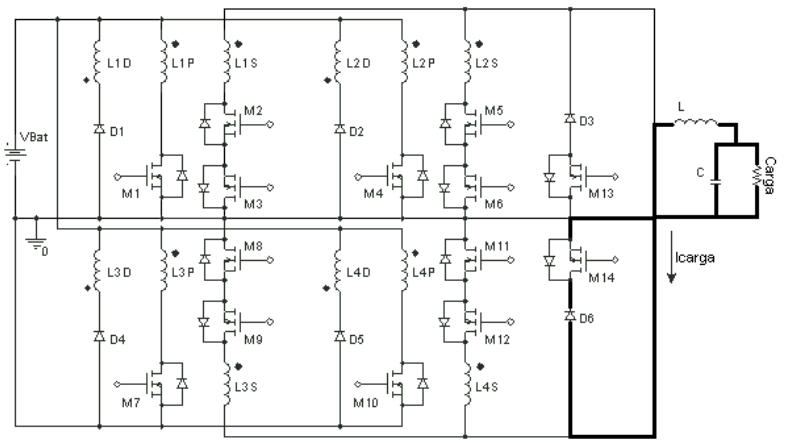

Figura 26. Décima Etapa

\section{Procedimento de Projeto}

Os parâmetros iniciais do projeto devem considerar a associação que é feita com os conversores para implementar o novo inversor.

A potência de saída pretendida do inversor é de $800 \mathrm{~W}$. Portanto, pode-se considerar que a potência de cada conversor será de $200 \mathrm{~W}$.

A tensão de entrada é de $12 \mathrm{~V}$ para todos os conversores. A tensão de saída do inversor será $110 \mathrm{~V}$ eficaz em tensão alternada. Para isso, é necessário um nível de tensão entregue na saída do transformador maior que o valor máximo entregue à carga. Com uma relação de espiras dos transformadores (n) de 0,05 , tem-se uma tensão de saída de $240 \mathrm{~V}$ no secundário, sendo $50 \%$ maior que o valor de pico de tensão entregue à carga, possibilitando assim, uma margem para compensar.

A corrente eficaz na saída do inversor será.

$$
\mathrm{Io}_{\mathrm{TOTAL}}=\frac{\mathrm{Ps}}{\mathrm{Vs}}=\frac{800}{110}=7,3(\mathrm{~A})
$$

Figura 25. Nona Etapa 
Portanto, a corrente eficaz no enrolamento secundário de cada conversor será.

$$
I s=\frac{I_{\text {TOTAL }}}{4}=1,85(A)
$$

Conseqüentemente, a corrente eficaz no enrolamento primário de cada conversor será.

$$
\text { Ip }=\frac{1,85}{0,05}=37(A)
$$

Considerando que a tensão de entrada varia entre $10 \mathrm{~V}$ e $14 \mathrm{~V}$ e Bmáx de $0,3 \mathrm{~T}$, o valor de B obtido é 0,21T Equação 1.10 (MELLO, 1990).

Considerando o parâmetro x igual 0,12 para núcleos do tipo EE (MELLO, 1990), o valor do parâmetro z é 1,14 Equação 1.8 (MELLO, 1990).

Com esses valores e considerando kj de 397, o valor do produto das áreas necessário para o projeto é de 3,72 cm $\mathrm{cm}^{4}$ Equação 1.9 (RASHID, 1993). Por meio da Tabela presente em Mello (1990), o menor núcleo que pode ser utilizado é o EE 42/21/15. Mas por disponibilidade do núcleo EE 42/21/20, este foi adotado.

Considerando o Dmáx igual a 0,5 , o número de espiras do primário é 5,0 Equação 1.11. E o número de espiras do secundário é 100 Equação 1.1 (MELLO, 1990).

O valor da indutância do primário é $0,12 \mathrm{mH}$ Equação 1.12 (MELLO, 1990). E a indutância do secundário é 47,5mH Equação 1.13 (MELLO, 1990).

Com o valor da indutância do enrolamento primário, o valor da corrente de magnetização obtido é de 2,92A Equação 1.16 (MELLO, 1990).

O valor da densidade de corrente para este núcleo é de 319,3 A/cm² Equação 1.17 (MELLO, 1990).

A área de cobre necessário para o primário é de $5,17.10^{-2} \mathrm{~cm}^{2}$. Para o secundário, é $5,64 \mathrm{e}-03 \mathrm{~cm}^{2}$. E para o desmagnetizante é $9,14 \cdot 10^{-3} \mathrm{~cm}^{2}$.
Com os valores das áreas de cobre necessárias para cada enrolamento, foi utilizado fita de cobre de 0,3mm x 25mm para o primário, 2 fios $20 \mathrm{AWG}$, em paralelo, para o secundário e 2 fios $20 \mathrm{AWG}$, também em paralelo, para o desmagnetizante. O fio $20 \mathrm{AWG}$ tem uma área de cobre igual a $5,18 \cdot 10^{-3} \mathrm{~cm}^{2}$.

O fio 20 AWG corresponde a um valor que pode ser utilizado na freqüência de $20 \mathrm{kHz}$, segundo a Figura 10.

Os valores dos componentes do filtro de saída devem ser calculados em função de freqüência de corte necessária. O cálculo da freqüência de corte e o valor da função de ripple são feitos com a Equação 1.19 (SEDRA; SMITH, 2000).

A freqüência de chaveamento dos transformadores dos conversores é igual a $20 \mathrm{kHz}$ e eles trabalham de forma a se complementarem dois a dois. Assim, a onda de tensão pulsada sobre o indutor tem uma freqüência de $40 \mathrm{kHz}$.

Para a escolha do núcleo a ser utilizado no indutor do filtro passa-baixa é necessário considerar a máxima energia que vai ser armazenada. Essa máxima energia ocorre nos picos de corrente de saída. Ao alimentar cargas com retificador em ponte completa na sua entrada, os picos de corrente podem ser dados pela Equação 4.4 (SEDRA; SMITH, 2000).

$$
i_{\text {Dmáx }}=I_{L}\left(1+2 \pi \sqrt{\frac{V_{p}}{2 \cdot V_{R}}}\right)
$$

Onde:

IDmáx - corrente de pico

Il - corrente eficaz

$\mathrm{Vp}$ - tensão de pico

$\mathrm{Vr}$ - tensão de pico a pico

Considerando a freqüência de $60 \mathrm{~Hz}$, tensão de pico de $110 \mathrm{~V}$, a carga de $21,1 \mathrm{~W}$ e a capacitância de $440 \mathrm{mF}$, o valor da tensão de pico a pico é facilmente encontrado pela Equação 4.5. 


$$
V_{R}=V_{P} \frac{1}{2 \cdot f \cdot C \cdot(4.5)}
$$

Para o projeto proposto, tem um valor de corrente de 41,53A.

Por meio da Equação 1.19 (SEDRA; SMITH, 2000) e utilizando um capacitor de $10 \mathrm{mF}$ com uma freqüência de corte de $3 \mathrm{kHz}$, encontra-se facilmente o valor do indutor de $280 \mathrm{mH}$.

Para este pico de corrente e esse valor de indutância, faz-se necessário a utilização de núcleo de ar, visto que o núcleo de ferrite não suportaria esse pico de energia.

Enrolando 180 espiras em um tubo de diâmetro de $2 \mathrm{~cm}$ com $9 \mathrm{~cm}$ de comprimento, chegou-se a indutância de $280 \mathrm{mH}$.

A área necessária de cobre para o indutor é de $22,86.10^{-3} \mathrm{~cm}^{2}$, levando-se em conta o efeito pelicular, pois tem-se a componente da corrente em alta freqüência. Isso corresponde a 14 fios de bitola 25 AWG. O fio 25 AWG tem uma área de cobre igual a $1,62.10^{-3} \mathrm{~cm}^{2}$ e pode ser utilizado com freqüências de $40 \mathrm{kHz}$, conforme indica a Figura 10.

\section{Resultados de Simulação e Experimentais}

Resultados de Simulações

Para simular o funcionamento da nova topologia de inversor de tensão, foi utilizado o software PSpice ${ }^{\circledR}$ AD 8.0. O circuito completo simulado é apresentado nas Figuras 27A, 27B 27C, 28 e 29.

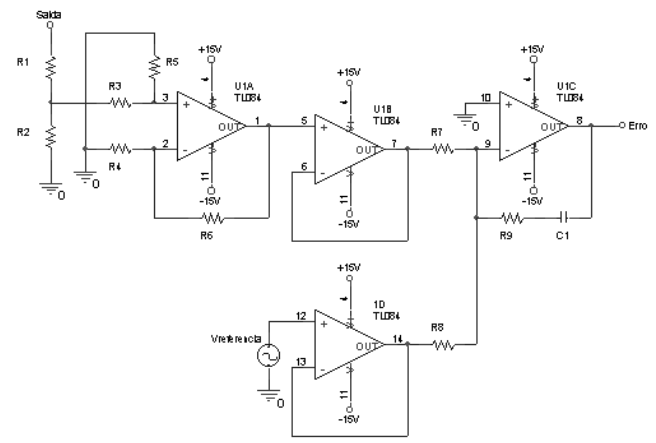

Figura 27A. Amplificador diferencial da saída e somador inversor com compensador integrador proporcional

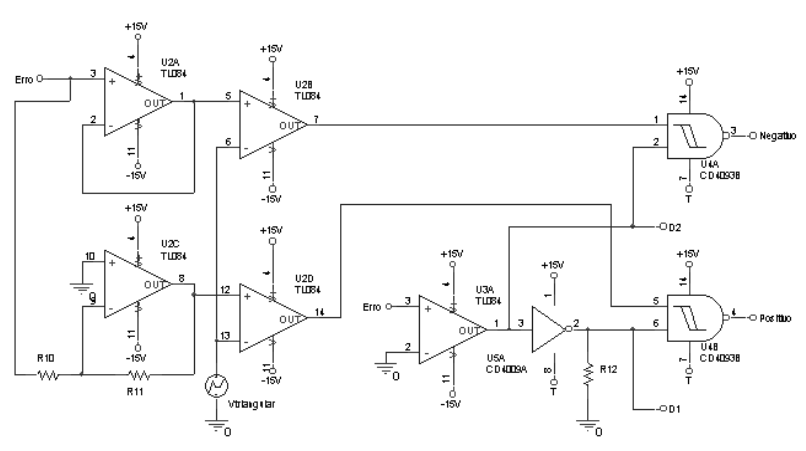

Figura 27B. Comparador e Separador de Pulsos

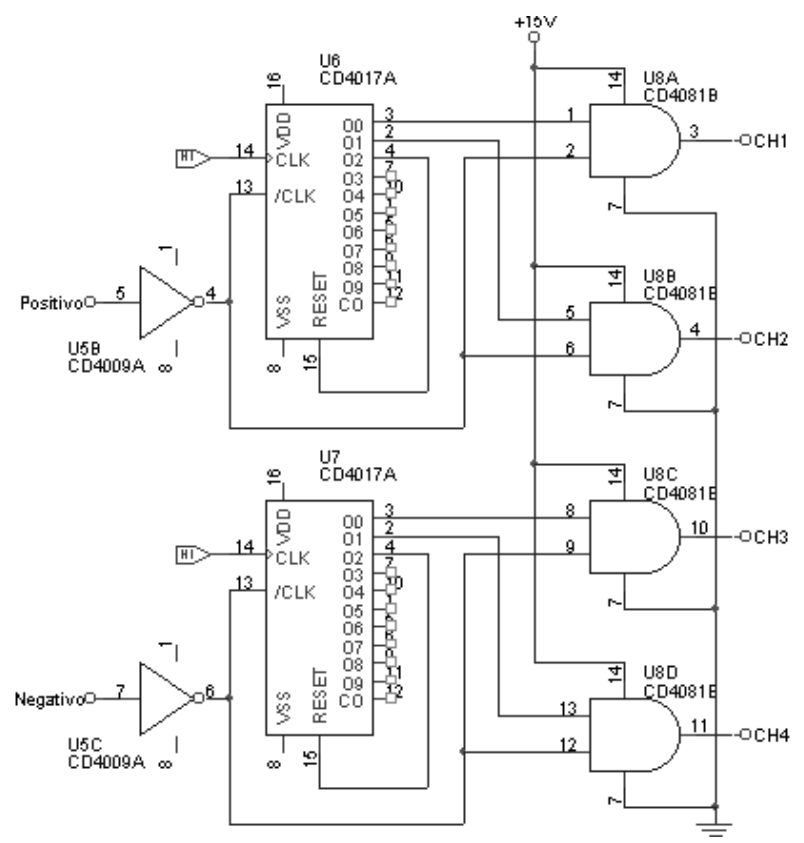

Figura 27C. Multiplexador

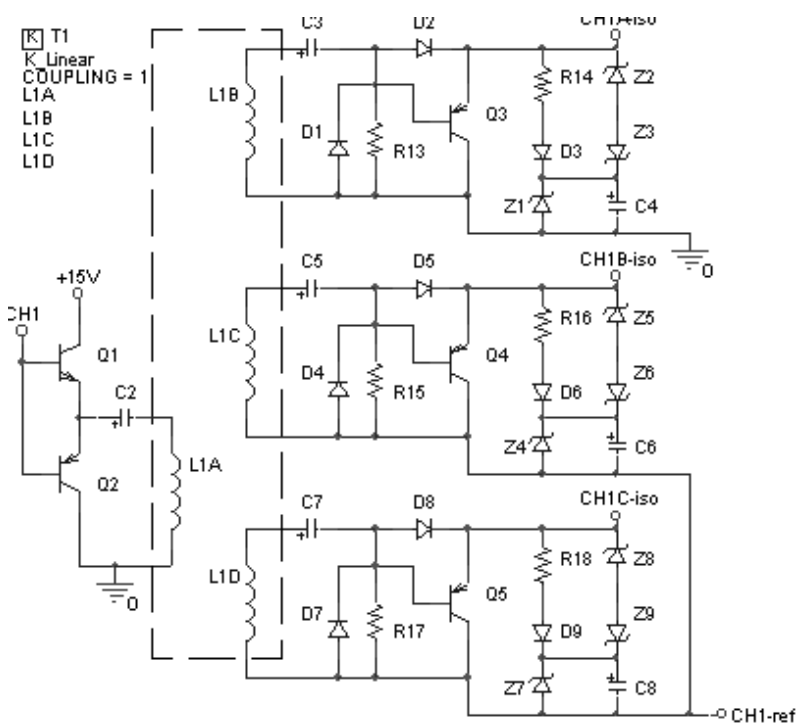

Figura 28. Isolador 


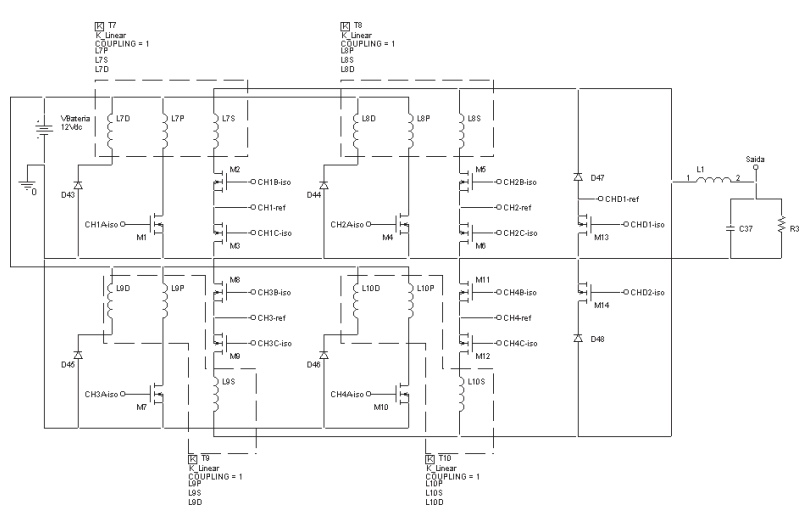

Figura 29. Circuito de Potência

Os parâmetros utilizados foram:

Tabela 2. Parâmetros de Simulação e Dispositivos Utilizados

\begin{tabular}{|c|c|c|}
\hline \multirow{3}{*}{ Primário do transformador } & \multirow{2}{*}{ Simulação } & \\
\hline & & Dispositivos Utilizados \\
\hline & $\mathrm{V}_{\text {DSmáx }}=24 \mathrm{~V} \mathrm{e} \mathrm{I}_{\mathrm{RMS}}=16,5 \mathrm{~A}$ & $\begin{array}{l}\text { IRFZ48N } \\
V_{D S}=55 \mathrm{~V} \text { e } I_{D}=64^{\mathrm{A}}\end{array}$ \\
\hline Secundário do Transformador & $\mathrm{V}_{\mathrm{DS} \text { máx }}=240 \mathrm{~V}$ e $\mathrm{I}_{\mathrm{RMS}}=1,8 \mathrm{~A}$ & $\begin{array}{l}\text { IRF740 } \\
V_{D S}=400 \mathrm{~V} \text { e } I_{D}=10^{A}\end{array}$ \\
\hline Diodos & $\mathrm{V}_{\text {DSmáx }}=240 \mathrm{~V}$ e $\mathrm{I}_{\mathrm{RMS}}=3,6 \mathrm{~A}$ & $\begin{array}{l}\text { IRF740 } \\
V_{D S}=400 \mathrm{~V} \text { e } I_{D}=10^{A}\end{array}$ \\
\hline Diodos de desmagnetização & $\mathrm{V}_{\mathrm{Rmáx}}=24 \mathrm{~V}$ e $\mathrm{I}_{\mathrm{RMS}}=2,9 \mathrm{~A}$ & $\begin{array}{l}\text { UF5404 } \\
V_{R}=400 V \text { e } I_{D}=3^{A}\end{array}$ \\
\hline Diodos de magnetização & $\mathrm{V}_{\mathrm{Rmáx}}=240 \mathrm{~V}$ e $\mathrm{I}_{\mathrm{RMS}}=3,6 \mathrm{~A}$ & $\begin{array}{l}\text { MUR850 } \\
V_{R}=500 \text { e }_{D}=8^{A}\end{array}$ \\
\hline
\end{tabular}

Também devido à freqüência de trabalho, todos os diodos são retificadores ultra-rápidos (Ultra-fast Recovery). A simulação produziu as formas de onda da Figura 30.

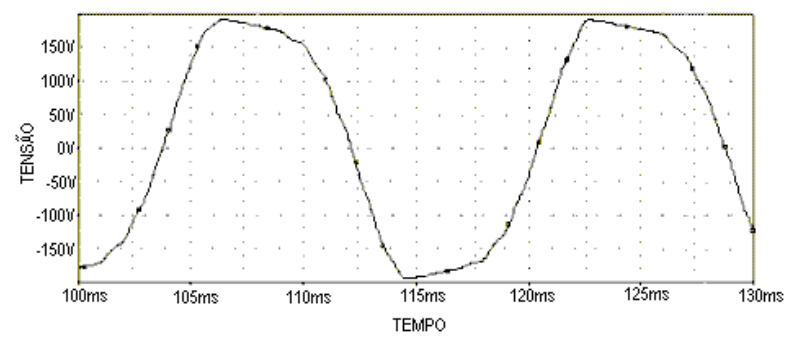

Figura 30. Tensão de saída da simulação

A Figura 30 mostra uma pequena distorção na forma de onda, devido a dificuldade de se ajustar as freqüências de corte do circuito de controle com a do circuito de potência.

\section{Resultados Experimentais}

Para a verificação do funcionamento da nova topologia de inversor de tensão, os circuitos apresentados nas Figuras 27A, 27B, 27C, 28 e 29 foram implementados.

O resultado dos testes é apresentado na Figura 31. É a onda presente na saída do conversor sobre uma carga resistiva.

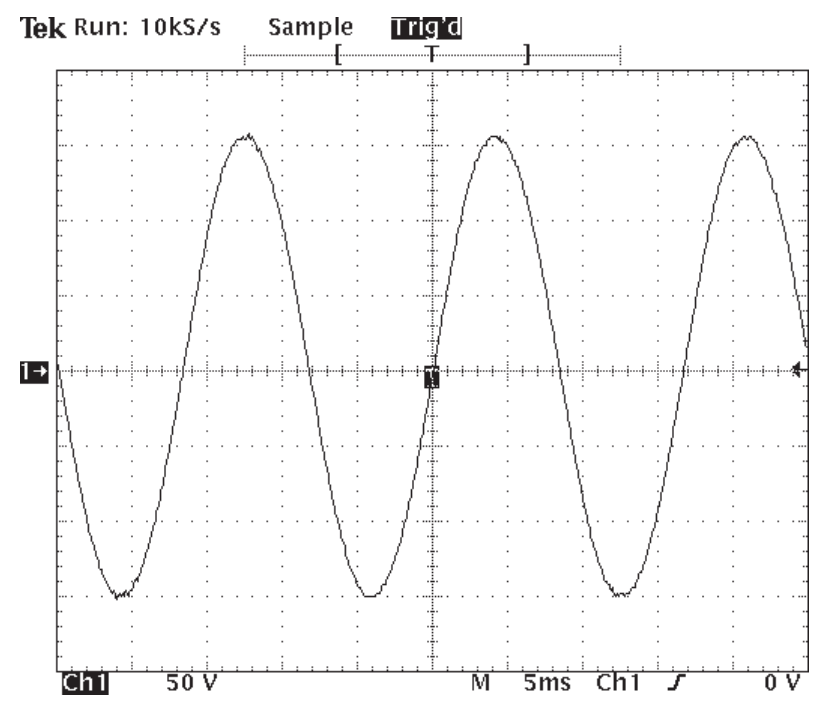

Figura 31. Tensão de saída do protótipo

Como se pode observar na Figura 31, a forma de onda é puramente senoidal, devido à implementação de uma malha fechada e ajustes do controle facilmente conseguido no laboratório, bastando trocar alguns capacitores.

\section{Fotografia do Protótipo}

Depois de verificados os resultados de simulação, foi implementado e testado em laboratório o protótipo do conversor com malha fechada, como mostrado na Figura 32. 


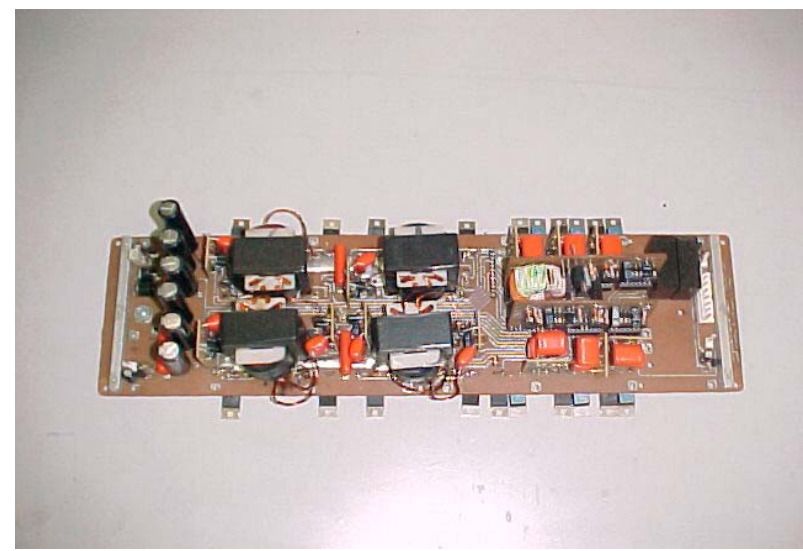

Figura 32. Foto do circuito implementado em laboratório

\section{Conclusão}

Neste trabalho foi apresentado uma nova topologia de inversor de tensão, utilizando transformador de alta freqüência e modulação PWM.

A simulação do circuito e os testes do protótipo produziram bons resultados, inclusive com baixo nível de ruído, apesar da necessidade de isolar um conversor do outro, aumentando o número de componentes e, conseqüentemente aumentando a complexidade do projeto.

Com a divisão de potência entre os quatro conversores forward, foi possível o projeto de um inversor de potência elevada $(800 \mathrm{~W})$ com núcleos magnéticos pequenos (EE 42/21/20).
Com a relação de transformação, o inversor proposto é capaz de compensar as quedas de tensão presentes na entrada, já que a tensão de saída do transformador apresenta um margem de compensação de $50 \%$ em relação àquela entregue à carga.

Esta topologia, composta por quatro conversores em paralelo, oferece um rendimento como uma estrutura de um único estágio de conversão, justificando sua aplicação comercial apesar de possuir um número considerável de chaves, sendo esta uma desvantagem, pois aumenta o custo do protótipo em torno de $30 \%$ se comparado à estrutura convencional. Devido ao seu rendimento e flexibilidade de projeto, esta proposta de inversão pode ser utilizada em sistemas de amplificadores de áudio.

\section{Referências}

MELLO, L. F. P. Análise e Projeto de Fontes Chaveadas. São Paulo: Editora Érica Ltda, 1990. 281p.

RASHID, M. H. Eletrônica de potência: circuitos, dispositivos e aplicações. 2.ed. São Paulo: Makron Books, 1993.

SEDRA, A. S.; SMITH, K. Circuitos de Microeletrônica. São Paulo: MAKRON Books, 2000.

TREVISO, C. H. G. Retificador de 6kW, Fator de Potência Unitário, Trifásico, Comutação Não-dissipativa na Conversão CC/CC e Controle Sincronizado em Freqüencia. Tese (Doutorado)- Uberlândia, 1999. 